\title{
On the inseparability of the Gauss map
}

\author{
Steven Kleiman ${ }^{1}$ and Ragni Piene ${ }^{2}$
}

\begin{abstract}
Let $X$ be a smooth complete intersection of degree at least 2, and consider these two new conjectures: (1) A general embedded tangent space is tangent at a unique point; in other words, the Gauss map is purely inseparable. (2) If $X$ is a hypersurface of degree at least 3 whose dual hypersurface is also smooth, then $X$ is either a plane cubic in characteristic 2 or the Fermat hypersurface with equation $\sum x_{i}^{q+1}=0$ where $q$ is a power of the characteristic. Both conjectures are known to hold if $X$ is a curve; in this paper, they are proved if $X$ is a surface, and supported if $X$ is an $n$-fold, $n \geq 3$. In addition, the separable degree of the Gauss map of an arbitrary projective curve $C$ is related to the number of cusps.
\end{abstract}

1. Introduction. Fix an algebraically closed ground field of characteristic $p$, and let $X$ be a smooth projective variety of degree $d$ at least 2 . Consider the Gauss map $\gamma$; it associates to each point $x$ of $X$ the point of the Grassmannian of $n$-planes that represents the embedded tangent space at $x$. It is well known that $\gamma$ is birational onto its image $X^{\prime}:=\gamma X$ in characteristic 0 , but sometimes not birational in positive characteristic. Over the last decade, there has been a substantial growth of interest in delineating and understanding the behavior of $\gamma$ in positive characteristic. The theorems proved, including those in this paper, suggest the following two conjectures:

ConJeCture 1. If $X$ is a complete intersection, then the Gauss map $\gamma$ is purely inseparable, or equivalently, generically injective.

Conjecture 2. If $X$ is a hypersurface of degree at least 3 whose dual hypersurface $X^{\prime}$ is also smooth, then $X$ is either a plane cubic in characteristic 2 or the Fermat hypersurface with equation $\sum x_{i}^{q+1}=0$ where $q=p^{e}$ for some $e \geq 1$.

If $X$ is a curve, Conjecture 1 is a consequence of an "inseparability theorem" proved by Kaji [16, Corollary 4.4, p. 187] (see also [21, Theorem, p. 1]), and Conjecture 2 is a theorem proved by Homma [13, 6.1, p. 1490 and 6.7, p. 1497] and reproved by Hefez [10, (7.18), p. 34].

In this paper, the conjectures are proved if $X$ is a surface (Theorems 13 and 14, the main results). Furthermore, the conjectures are supported if $X$ is an $n$-fold and $X^{\prime}$ is smooth, with the following two results, which the conjectures would

\footnotetext{
${ }^{1}$ Supported in part by NSF grant 8801743 DMS and in part by the Danish Natural Science Research Council.

${ }^{2}$ Supported in part by a fellowship at the Bunting Institute and in part by the Norwegian Research Council for Science and the Humanities.

1980 Mathematics subject classifications. Primary 14N05; Secondary 14N10, 14 M10.
}

To appear in the Proceedings of the 1989 Zeuthen Symposium Comtemporary Mathematics, American Mathematical Society 
imply respectively: (1) (Theorem 4) if $X$ is a complete intersection, then $X$ and $X^{\prime}$ have the same top Chern class; and (2) (Theorem 5) if $X$ is a hypersurface of degree $d$ at least 3 , then $X^{\prime}$ is also of degree $d$, and $\gamma$ is of degree $(d-1)^{n}$. Moreover, if $X$ is a smooth hypersurface of degree 2, then the following result (Proposition 6) is proved without assuming that $X^{\prime}$ is smooth: if $n$ is odd and the characteristic is 2 , then $X^{\prime}$ is a hyperplane and $\gamma$ is purely inseparable of degree 2 ; otherwise, $X^{\prime}$ too is a smooth quadric and $\gamma$ is an isomorphism. Consequently, (Theorem 7) if $X$ is a hypersurface whose tangents contain a common point, $X$ is an odd dimensional quadric in characteristic 2 , and conversely. In addition, the Gauss rational map of an arbitrary projective curve $C$ is studied. Specifically, the following two links are made among the separable degree $s$, the number of cusps $\kappa$, and the geometric genera $g$ and $g^{\prime}$ of $C$ and its image (Theorem 8): (1) $2 s\left(g-g^{\prime}\right) \leq(s-1) \kappa$; and (2) if $\kappa<2 g-2$, then $s=1$. Inequality (1) is illustrated with two examples (Example 10); in the first, equality holds, and in the second, equality fails.

In arbitrary characteristic, the Gauss map $\gamma$ of $X$ is finite by a theorem of Zak's $[6,7.2$, p. 58] because $X$ is smooth; that theorem has a simple proof if $X$ is a complete intersection, see Lemma 2. (Over the complex numbers, Griffiths and Harris [9, (2.29), p. 393] gave a proof that $\gamma$ is generically finite, and they mentioned two other proofs in a footnote; generic finiteness suffices in what follows.) Since $\gamma: X \rightarrow X^{\prime}$ is (generically) finite, it is birational if a general fiber $F$ is scheme-theoretically a linear space. In characteristic $0, F$ is automatically reduced, and it is known that $F$ is set-theoretically a linear space (whether $X$ is smooth or not). That fact was proved by Zak according to Fulton and Lazarsfeld $[6$, Note (1), p. 66]; it too was proved over the complex numbers by Griffiths and Harris [9, (2.10), p. 388]. Thus $\gamma: X \rightarrow X^{\prime}$ is birational in characteristic 0 .

In arbitrary characteristic, $\gamma: X \rightarrow X^{\prime}$ is birational if $X$ is reflexive, that is, if $C X=C X^{*}$ where $C X$ is the conormal variety and $X^{*}$ is the dual variety. Indeed, again, because $\gamma$ is finite, it suffices to prove that (whether or not $X$ is smooth) a general fiber $F$ of $\gamma$ is scheme-theoretically a linear space. To prove it, consider the following diagram with Cartesian square:

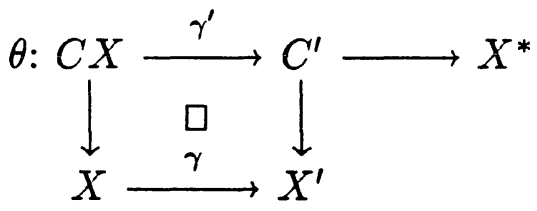

where $C^{\prime}$ is the restriction over $X^{\prime}$ of the appropriate tautological bundle of projective spaces over the Grassmannian. Note that $C^{\prime}$ parametrizes the pairs consisting of a tangent $n$-plane and a hyperplane containing it. (In connection with that diagram, there is an interesting open question. In the case that $X$ is a curve, but not necessarily smooth nor reflexive, Voloch [24, Theorem 1] observed that $\theta$ and $\gamma$ have the same inseparable degree because of [11, Theorem 3.5] and [12, Proposition 4 and the following remark]; since the square is Cartesian, it is equivalent to say that $C^{\prime} \rightarrow X^{*}$ is separable. Is $C^{\prime} \rightarrow X^{*}$ separable in higher dimension?)

To finish the discussion of $F$, say $F:=\gamma^{-1} T$ where $T$ is a general point of $X^{\prime}$, and let $H$ be a hyperplane containing the (n-plane) $T$. Because the square 
is Cartesian, $F$ is equal to the fiber of $\gamma^{\prime}$ over the point $(T, H)$ of $C^{\prime}$; hence, $F$ is contained in $\theta^{-1} H$ scheme-theoretically. If $H$ is a smooth point of $X^{*}$, then $\theta^{-1} H$ is a linear space because $X$ is reflexive. Hence, $F$ is scheme-theoretically contained in the intersection $G:=\bigcap \theta^{-1} H$, which is a linear space. On the other hand, if a point $x$ of $X$ does not lie in $F$, then its tangent space $T_{x}$ is not equal to $T$; hence, a general hyperplane $H$ containing $T$ does not contain $T_{x}$; hence, $x \notin \theta^{-1} H$. Therefore, $F=G$ as sets, whence as schemes because $G$ is reduced. Thus the scheme $F$ is a linear space, as asserted.

In arbitrary characteristic, $X$ is reflexive, according to the Monge-SegreWallace criterion $\left[20,(4)\right.$, p. 169], if and only if $\theta: C X \rightarrow X^{*}$ is separable. In characteristic 0 , therefore, $X$ is reflexive. Thus, in characteristic 0 , the argument above yields another proof that the scheme $F$ is a linear space, and so another proof that $\gamma: X \rightarrow X^{\prime}$ is birational. In arbitrary characteristic, most $X$ are reflexive; for example, a general complete intersection is reflexive, except if it is of odd dimension in characteristic $2[11,(5.6)]$. Thus Conjecture 1 holds if $X$ is general; in fact, then $\gamma: X \rightarrow X^{\prime}$ is birational.

In positive characteristic $p$, the Gauss map $\gamma: X \rightarrow X^{\prime}$ is sometimes not birational. Wallace $[\mathbf{2 5}, 7.2$, p. 340] in 1956 gave the first example of such an $X$, the Fermat curve of degree $p+1$. The situation is the same for the $n$-dimensional Fermat hypersurface $X$ of degree $q+1$, where $q=p^{e}$ for some $e \geq 1$. In a suitable coordinate system, $X$ has the equation $\sum x_{i}^{q+1}=0$. Obviously, $\gamma$ may be identified with the Frobenius $q$ th power map. Hence, $\gamma$ is purely inseparable of degree $q^{n}$, and $X^{\prime}\left(=X^{*}\right)$ is smooth of the same degree, $q+1$, as $X$. Although the identification of $X^{\prime}$ with $X$ depends on the choice of coordinates, the resulting identification of $X^{\prime \prime}$ with $X$ is canonical, where $X^{\prime \prime}$ is the dual of $X^{\prime}$. Note that $X$ is not reflexive, as the composition of the two Gauss maps is not equal to the identity of $X$. In this connection, Garcia and Voloch recently proved a lovely theorem $[8$, Theorem 4 , p. 17]: let $X$ be a nonreflexive, smooth plane curve of degree at least 4. Then, $X^{\prime \prime}=X$ if and only if $X$ is "Frobenius nonclassical"; that is, $X$ is defined over the field with $q:=p^{e}$ elements for some $e$ and, for each point $x$, the tangent line at $x$ contains the image of $x$ under the Frobenius $q$ th power map. Hefez and Voloch made a general study of Frobenius nonclassical curves in [12], and Garcia gave examples of smooth ones other than the Fermat curve in [7]. Does Garcia and Voloch's theorem generalize to higher dimension?

Conjecture 1 holds if $X$ is a smooth curve of degree 2 or 3 , whether $X$ is a complete intersection or not. Indeed, if a general fiber of $\gamma$ contained two distinct points, then a general hyperplane containing them would be tangent at each of them, and Bezout's theorem would be contradicted.

In characteristic 2, the Gauss map $\gamma$ of every variety of odd dimension $n$ is of inseparable degree at least 2 ; that result was proved by N. Katz [18, Proposition 3.3, p. 221, and $\S 1.2$, p. 214]. Hence, if $X$ is a smooth plane cubic, then so is the dual curve $X^{\prime}$, as allowed by Conjecture 2. Indeed, the degree $m$ of $\gamma$ is at least 2, and the degree $d$ of $X$ is equal to 3, so the degree $d^{\prime}$ of $X^{\prime}$ is at most 3 thanks to the well-known Plücker formula $m d^{\prime}=d(d-1)^{n}$, compare with (3.4). Moreover, $\gamma$ is purely inseparable because $X$ is of degree 3 (as was noted above); so the geometric genus of $X^{\prime}$ is also 1 . Hence $X^{\prime}$ is also a smooth cubic, 
as claimed. However, if $X$ is a smooth cubic 3 -fold in 4-space, then $X^{\prime}$ need be neither smooth nor a cubic. For example, consider

$$
X: x_{0}^{3}+x_{0} x_{1}^{2}+x_{0} x_{1} x_{3}+x_{2}^{3}+x_{3}^{3}+x_{4}^{3}=0 .
$$

A computation, carried out using the computer algebra program MACAULAY, yields this: $X^{\prime}$ is of degree 12; its singular locus is of dimension 2 and degree 72. On the other hand, the Plücker formula now implies that $\gamma: X \rightarrow X^{\prime}$ is of degree $m=2$; so $\gamma$ is purely inseparable, in keeping with Conjecture 1.

The first examples of a smooth $X$ such that $\gamma: X \rightarrow X^{\prime}$ is inseparable, but not purely inseparable, were given independently by Kaji $[15,4.1$, p. 439] and Rathmann [23, 2.13, p. 576]. Both found suitable curves of genus 0, and later Kaji $[16,0.1$, p. 177] found ones of genus 1 . Note that none of these curves is a complete intersection, so none is a counterexample to Conjecture 1 . Indeed, as noted above, none is of degree 2 or 3 . On the other hand, a smooth complete intersection of degree at least 4 is of genus $g$ at least 1 , and if $g=1$, then the curve is an elliptic quartic on a quadric surface. Hence, if $g=1$, then the curve has no bitangent, so its Gauss map is purely inseparable.

Kaji's original inseparability theorem dealt not simply with a smooth curve, but more generally with an immersed curve (one with simple branches), and asserted this: if the geometric genus $g$ is at least 2, then the Gauss rational map is purely inseparable. That assertion is generalized in Theorem 8 to a curve $C$ with arbitrary singularities, provided $\kappa<2 g-2$ where $\kappa$ is the number of cusps; more precisely, $\kappa:=\operatorname{deg} \Omega_{X / C}^{1}$ where $X$ is the normalization. Independently, Kaji [17] found the same generalization, and he gave examples showing that the bound $2 g-2$ is optimal. Kaji's original proof, his second proof, and the proof of Theorem 8 are all a little different. The proof of Theorem 8 also gives the inequality, $2 s\left(g-g^{\prime}\right) \leq(s-1) \kappa$, where $s$ is the separable degree of the Gauss map and $g^{\prime}$ is the geometric genus of its image. In fact, the proof yields a little more: equality holds if each singularity of $C$ is an ordinary multiple point or, except in characteristic 2, a simple cusp. That case is illustrated in Example 10 with an interesting example of Bayer and Hefez's. Also, the case of strict inequality is illustrated with a simple example of Hefez's.

Kaji's original work [16] on inseparability broke ground not only with the theorem itself, but also with the method of proof. The idea is to do geometry on the total space $Y^{\prime}$ of a certain natural family of tangent lines, namely, the family parametrized by the normalized image $X^{\prime}$ of the Gauss map. Thus, if $Y$ is the total space of the family parametrized by the normalization $X$ of $C$, then there is a natural finite map $Y \rightarrow Y^{\prime}$, and its degree is equal to the degree $m$ of the Gauss map. Two curves play a key role: the canonical section $S$ of $Y / X$, and its image $D^{\prime}$ in $Y^{\prime}$. Kaji determined the canonical class on $Y^{\prime}$, and then the arithmetic genus of $D^{\prime}$, finding $p_{a}\left(D^{\prime}\right)=m\left(g^{\prime}-g\right)+g$. Since $p_{a}\left(D^{\prime}\right) \geq g \geq g^{\prime}$, therefore $g=g^{\prime}$. Finally, the Riemann-Zeuthen-Hurwitz formula implies that, when the Gauss map is not purely inseparable, then $g>g^{\prime}$, because $g^{\prime} \geq 2$ as $g \geq 2$ by hypothesis.

In this paper, the leading new idea is to compute the self-intersection number of the cycle $[S]$ on $Y$ in two ways. First, since $S$ is a section of $Y / X$ such that 
$\mathcal{O}_{Y}(1) \mid S$ is equal to $O_{X}(1)$, it is not hard to prove that $[S]^{2}=\kappa-2 g+2$; see (8.4). Secondly, Lemma 2 asserts that $s[S]$ is numerically equivalent to $[D]$, where $D$ is defined as follows. Replace $Y^{\prime}$ by its normalization in the purely inseparable closure of its function field in function field of $Y$, and then define $D$ as the inverse image of $D^{\prime}$, where as before $D^{\prime}$ denotes the image of $S$ in $Y^{\prime}$. Since $Y / Y^{\prime}$ is separable, the cycle $[S]$ appears with multiplicity 1 as a component of $[D]$. Hence, since $Y$ is a surface, the effective cycle $[D]-[S]$ meets $[S]$ properly. Therefore, $(s-1)[S]^{2} \geq 0$. Consequently, if $s>1$, then $\kappa \geq 2 g-2$. Now, a little additional computation shows that $2\left(p_{a}\left(D^{\prime}\right)-g\right)$ is equal to $(s-1) \kappa-2 s\left(g-g^{\prime}\right)$; see (8.6), and see Remark 9 for two variations on the computation. Therefore, $2 s\left(g-g^{\prime}\right) \leq(s-1) \kappa$.

Suppose that $X$ is a smooth complete intersection of degree $d$ at least 2 and of arbitrary dimension $n$, and that the image $X^{\prime}$ of the Gauss map $\gamma$ is also smooth. Then similar considerations apply to some extent. First, $[S]^{2}$ is equal to the top Chern class $^{3} c_{n}(X)$, and $\left[D^{\prime}\right]^{2}$ is equal to $m c_{n}\left(X^{\prime}\right)$ where $m:=\operatorname{deg}(\gamma)$. Consequently, the following equation holds, see Theorem 4: $c_{n}(X)=c_{n}\left(X^{\prime}\right)$. That equation suggests that $\gamma$ is purely inseparable, because a purely inseparable map is an isomorphism in the étale topology, and the top Chern class is equal to the topological Euler characteristic.

Suppose that $X$ is a smooth hypersurface of degree at least 2. Then $c_{n}(X)$ is given by a polynomial $P(d)$; moreover, $(-1)^{n} P(d)$ is strictly increasing in $d$ for $d \geq 2$, and $P(2)>P(1)$ if $n$ is even, but $P(2)=P(1)$ if $n$ is odd. Theorem 5 follows, asserting this: if $X^{\prime}:=\gamma X$ is also smooth, then $X^{\prime}$ is also of degree $d$, and $\gamma$ is of degree $(d-1)^{n}$ with this one exception, $X$ is an odd-dimensional quadric in characteristic 2 and $X^{\prime}$ is a hyperplane. If $X$ is the Fermat hypersurface of degree $d=q+1$ with $q=p^{e}$ and $e \geq 1$, then, as noted above, it is easy to check the conclusion of Theorem 5 directly; thus, Theorem 5 supports Conjecture 2.

Suppose that $X$ is a smooth hypersurface of degree at least 2. Then Theorem 7 asserts this: a necessary and sufficient condition that the tangent hyperplanes of $X$ contain a common point is that $X$ be an odd dimensional quadric in characteristic 2 . The necessity results immediately from Theorem 5 ; the sufficiency is asserted by Proposition 6 . The case where $X$ is a smooth plane curve is part of a celebrated 1962 theorem of Lluis, who proved it for nodal (or immersed) curves in $N$-space. (Lluis's theorem was rediscovered for smooth curves by Samuel in 1966, and Samuel is often credited with the result.) The general case of Theorem 7 was conjectured by Hefez and Levcovitz (pvt. comm., 16 April 1990), who were on the track of a different proof.

Suppose that $X$ is a smooth surface and a complete intersection of degree $d$ at least 2. Then, although $[S]$ does still appear with multiplicity 1 as a component of $[D]$, conceivably the cycle $[D]-[S]$ no longer meets $[S]$ properly. That possibility is handled as follows. Form the blowup $\tilde{Y}$ of $Y$ along $S$, and the strict transform $\widetilde{S}$ of $D$. Then compute the refined intersection number $[S] \bullet_{Y}[\widetilde{S}]$ in two ways. First, the number is equal to $(s-1) c_{2}(X)$ because $[D]$ is numerically equivalent to $s[S]$. Secondly, compute the number by using the excess-intersection formula

\footnotetext{
${ }^{3}$ In this paper, surfaces are of primary interest, and for them, that invariant was discovered in 1871 by Zeuthen. It, diminished by 4 , was rediscovered in 1894 by C. Segre, and is still called the Zeuthen-Segre invariant.
} 
and considering a certain natural map from $\tilde{Y}$ to the Grassmannian of lines. Combined, the two computations yield an expression for $(s-1)\left(c_{2}(X)-4 d\right)$ as a sum of two nonpositive terms; see Proposition 11, which is set in a slightly more general context. Now, $c_{2}(X)-4 d>0$, whence $s=1$, except in five cases; see Lemma 12. Those five cases can be treated directly. Thus, in any case, $s=1$, as asserted by the first main theorem, Theorem 13 .

Finally, suppose that $X$ is a smooth surface of degree $d$ at least 3 in 3-space, and that the dual surface $X^{\prime}$ is smooth too. The results so far imply that $X^{\prime}$ is also of degree $d$ and that $\gamma: X \rightarrow X^{\prime}$ is purely inseparable of degree $(d-1)^{2}$. So $d=q+1$ with $q:=p^{e}$ for some $e \geq 1$. Let $C^{\prime}$ be a general hyperplane section of $X^{\prime}$, and $C$ its reduced inverse image $\left(\gamma^{-1} C^{\prime}\right)_{\text {red }}$ on $X$. An argument shows that $C$ is nonsingular, and then that it is a hyperplane section of $X$; moreover, $\gamma^{-1} C^{\prime}=q C$. By definition, $\gamma^{-1} C^{\prime}$ is a general first polar of $X$. Hence, the linear system of all first polars is equal to the $q$ th multiple of the linear system of plane sections. Therefore, by a lovely theorem of Beauville's [2], $X$ is the Fermat hypersurface, as asserted by the second main theorem, Theorem 14 .

2. The top Chern class. The main result of this section, Theorem 4 , concerns a smooth complete intersection $X$ of arbitrary dimension $n$. It asserts that, if the image $X^{\prime}$ of the Gauss map is smooth too, then $X$ and $X^{\prime}$ have the same $n$th Chern class. However, the key lemma in the proof, Lemma 2, will be applied in the next section to a different sort of $X$, namely, the normalization of a given projective curve $C$. For that reason, $X$ is not initially taken to be embedded in the ambient projective space, let alone to be a complete intersection.

Setup 1. Let $X$ be a smooth $n$-dimensional variety (reduced and irreducible algebraic scheme) equipped with a finite birational map $\phi: X \rightarrow C$, where $C$ is a closed subvariety of a projective space $\mathbf{P}(V)$, and $V$ is a finite dimensional vector space. Set

$$
\mathcal{L}:=\phi^{*} \mathcal{O}_{C}(1)
$$

Let $\mathcal{P}$ be the image of the canonical map $a_{1}: V_{X} \rightarrow \mathcal{P}_{X}^{1}(\mathcal{L})$ where $\mathcal{P}_{X}^{1}(\mathcal{L})$ is the sheaf of principal parts of sections of $\mathcal{L}$ (see [22, pp. 252-253] or [19, pp. 342346]). Assume that $\mathcal{P}$ is locally free, or equivalently, that the Gauss map of $C$ is defined everywhere on $X$. By definition, the target of the Gauss map is the Grassmannian of $n$-planes in $\mathbf{P}(V)$. Algebraically, the map corresponds to the canonical surjection $V_{X} \rightarrow \mathcal{P}$; that is, $\mathcal{P}$ is equal to the pullback of the universal quotient sheaf. Geometrically, the map is defined by sending a point $x$ of $X$ at which $\phi$ is an isomorphism to the embedded tangent space $T_{\phi x}$ of $C$.

Consider the abstract tangent developable $Y$; by definition,

$$
Y:=\mathbf{P}(\mathcal{P})
$$

Thus, $Y$ is equal to the pullback, under the Gauss map, of the incidence variety $I$ of points and $n$-planes. Let $\pi: Y \rightarrow X$ denote the structure map. It has a natural section $\sigma: X \rightarrow Y$. Indeed, the canonical surjection $V_{X} \rightarrow \mathcal{L}$ factors through $\mathcal{P}_{X}^{1}(\mathcal{L})$, so through $\mathcal{P}$, and the induced surjection $\mathcal{P} \rightarrow \mathcal{L}$ defines $\sigma$. By virtue of that definition,

$$
\mathcal{L}=\sigma^{*} \mathcal{O}_{Y}(1)
$$


In geometric terms, $\sigma$ may be described as follows: Let $x$ be a point of $X$ at which $\phi$ is an isomorphism, and identify the fiber $\pi^{-1} x$ with the embedded tangent space $T_{\phi x}$. Then $\sigma x$ is equal to $\phi x$ viewed as a point of $T_{\phi x}$.

Consider the image of the section $\sigma$,

$$
S:=\sigma X
$$

By the self-intersection formula [5, Cor. 6.3, p. 102],

$$
[S]^{2}=c_{n}\left(\nu_{\sigma}\right)
$$

where $\nu_{\sigma}$ is the normal sheaf of $\sigma$ (or what is the same, that of $S$ ). If $\tau_{\pi}$ is the (relative) tangent sheaf, then $\nu_{\sigma}$ is given by the well-known formula,

$$
\nu_{\sigma}=\sigma^{*} \tau_{\pi}
$$

(That formula holds because $\sigma$ is a base extension over itself of the diagonal map of $\pi$, and the formation of the normal sheaf of a flat subscheme commutes with base change.) There is a second useful formula for $\nu_{\sigma}$,

$$
\nu_{\sigma}=\Omega^{*}
$$

where $\Omega^{*}$ is the dual of the sheaf defined by the natural exact sequence,

$$
0 \longrightarrow \Omega \otimes \mathcal{L} \longrightarrow \mathcal{P} \longrightarrow \mathcal{L} \longrightarrow 0
$$

Indeed, see $[\mathbf{1},(2.6)(\mathrm{i}), \mathrm{pp} .17]$, the ideal of $S$ in $Y$ is equal to the image of the natural map,

$$
\left(\pi^{*}(\Omega \otimes \mathcal{L})\right) \otimes \mathcal{O}_{Y}(-1) \longrightarrow \mathcal{O}_{Y}
$$

because of the following sequence, whose exactness is standard [4, Ch. III, $\S 6$, no. 2, Prop. 4, p. 499]:

$$
(\Omega \otimes \mathcal{L}) \operatorname{Sym}(\mathcal{P})[-1] \longrightarrow \operatorname{Sym}(\mathcal{P}) \longrightarrow \operatorname{Sym}(\mathcal{L}) \longrightarrow 0
$$

Hence there is a surjection,

$$
\sigma^{*}\left(\left(\pi^{*}(\Omega \otimes \mathcal{L})\right) \otimes \mathcal{O}_{Y}(-1)\right) \longrightarrow \nu_{\sigma}^{*}
$$

That surjection is an isomorphism because its source and target are locally free of the same rank (namely, $n$ ). Finally, (1.1) yields (1.4).

Let $\gamma: X \rightarrow X^{\prime}$ be a surjective map, and suppose that the Gauss map of $X$ factors through $\gamma$. Let $Y^{\prime}$ be the pullback to $X^{\prime}$ of the incidence variety $I$. Then there is a diagram with Cartesian square

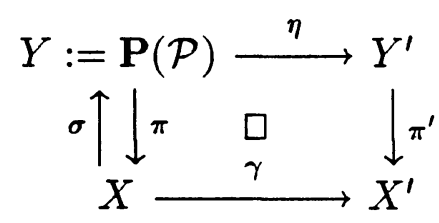


where $\pi^{\prime}$ and $\eta$ are the natural maps. Note that $Y^{\prime}:=\mathbf{P}\left(\mathcal{P}^{\prime}\right)$ where $\mathcal{P}^{\prime}$ is the pullback to $X^{\prime}$ of the universal quotient sheaf on the Grassmannian.

Set $\sigma^{\prime}=\eta \sigma$, and form the following closed subvariety of $Y^{\prime}$ :

$$
D^{\prime}:=\sigma^{\prime} X=\eta S
$$

Then the map $X \rightarrow D^{\prime}$ is birational; indeed, the composition $Y^{\prime} \rightarrow I \rightarrow \mathbf{P}(V)$ carries $D^{\prime}$ to $C$, and the composition $X \rightarrow D^{\prime} \rightarrow C$ is equal to $\phi$. Moreover, if $\phi=1_{X}$, then $X \rightarrow D^{\prime}$ is an isomorphism; in other words, $\sigma^{\prime}$ is an embedding. Since $X \rightarrow D^{\prime}$ is birational, $D^{\prime}$ is of dimension $n$.

Finally, form another closed subscheme $D$ of $Y$ by discarding the embedded components of $\eta^{-1} D^{\prime}$; in other words, form $D$ as the closure of the open subscheme of $\eta^{-1} D^{\prime}$ whose complement is the union of the embedded components. Obviously, $[D]=\left[\eta^{-1} D^{\prime}\right]$. Note that $D$ is reduced if $\gamma$ is separable because $\eta^{-1} D^{\prime}$ is equal to $X \times_{X^{\prime}} D^{\prime}$ and both $\gamma: X \rightarrow X^{\prime}$ and $D^{\prime} \rightarrow X^{\prime}$ are surjective maps of varieties (and because, if $L / K$ and $M / K$ are two field extension and if $L / K$ is separable, then the ring $L \otimes_{K} M$ is reduced).

Suppose $\gamma$ is finite. Then so is $\eta$. Hence $D$ is of pure dimension $n$. Suppose in addition that $\gamma$ is flat. Then so is $\eta$. Hence $\eta^{*}\left[D^{\prime}\right]$ is equal to $\left[\eta^{-1} D^{\prime}\right]$, so to $[D]$. Therefore, the projection formula yields

$$
[D]^{2}=m\left[D^{\prime}\right]^{2} \text { where } m:=\operatorname{deg} \gamma
$$

because $\eta$ and $\gamma$ are of the same degree.

LEMmA 2. Under the conditions of Setup 1, assume that there is an invertible sheaf $\mathcal{L}^{\prime}$ on $X^{\prime}$ and a nonzero rational number a such that $c_{1}\left(\gamma^{*} \mathcal{L}^{\prime}\right)$ is numerically equivalent to $a c_{1}(\mathcal{L})$. Then $\gamma$ is finite, and the $n$-cycles $[D]$ and $m[S]$ on $Y$ are numerically equivalent, where $m:=\operatorname{deg} \gamma$.

Proof. If $\gamma$ were not finite, then one of its fibers would contain a complete curve $A$. Hence, the hypothesis would yield the following contradiction:

$$
0=c_{1}\left(\gamma^{*} \mathcal{L}^{\prime}\right)[A]=a c_{1}(\mathcal{L})[A] \neq 0
$$

Set $k:=c_{1}\left(\mathcal{O}_{Y}(1)\right)$. Then the classes of codimension $n$ on $Y:=\mathbf{P}(\mathcal{P})$ are generated by the products $k^{j} \pi^{*} z$ where $0 \leq j \leq n$ and $z$ ranges over the classes of codimension $n-j$ on $X$. Hence, to prove that $[D]$ and $m[S]$ are numerically equivalent, it suffices to prove that

$$
k^{j} \pi^{*} z([D]-m[S])=0 \text {. }
$$

Since $X \rightarrow D^{\prime}$ is birational, $D^{\prime} \rightarrow X^{\prime}$ is, like $\gamma: X \rightarrow X^{\prime}$, a finite surjective map of degree $m$ between varieties. Hence, clearly, $D$ is equal to the closure of the product over $X^{\prime}$ of a suitably small dense open subset of $X$ with one of $D^{\prime}$. Therefore, $D \rightarrow X$ is a finite surjective map of degree $\mathrm{m}$. Consequently,

$$
\pi_{*}[D]=m[X]
$$


The classes $a c_{1}\left(\mathcal{O}_{Y^{\prime}}(1)\right) \mid D^{\prime}$ and $\left.c_{1}\left(\pi^{\prime *} \mathcal{L}^{\prime}\right)\right) \mid D^{\prime}$ are numerically equivalent, because their pullbacks to $X$ are numerically equivalent by (1.1) and by hypothesis, and because the map $X \rightarrow D^{\prime}$ is surjective. Hence their pullbacks under $D \rightarrow D^{\prime}$ are numerically equivalent on the scheme $D$. Those pullbacks are equal to $a k \mid D$ and $\pi^{*} c_{1}\left(\gamma^{*} \mathcal{L}^{\prime}\right) \mid D$ respectively.

Since $a k \mid D$ and $\pi^{*} c_{1}\left(\gamma^{*} \mathcal{L}^{\prime}\right) \mid D$ are numerically equivalent, the projection formula yields

$$
\begin{aligned}
(a k)^{j} \pi^{*} z([D]-m[S]) & =\pi^{*}\left(c_{1}\left(\gamma^{*} \mathcal{L}^{\prime}\right)^{j} z\right)([D]-m[S]) \\
& =c_{1}\left(\gamma^{*} \mathcal{L}^{\prime}\right)^{j} z \pi_{*}([D]-m[S])
\end{aligned}
$$

Since $\sigma$ is a section, $\pi_{*}[S]=[X]$. Hence (2.2) implies that $\pi_{*}([D]-m[S])=0$. Therefore (2.1) holds. Thus the lemma is proved.

Setup 3. Under the conditions of Setup 1, suppose that $X$ is embedded in $\mathbf{P}(V)$ (and that $\left.\phi=1_{X}\right)$. Then $\mathcal{P}=\mathcal{P}_{X}^{1}(\mathcal{L})$, and $\Omega=\Omega_{X}^{1}$ because of $(1.5)$; so $(1.4)$ yields

$$
\nu_{\sigma}=\tau_{X}
$$

Let $\nu_{X}$ be the normal sheaf, and $\nu_{X}^{*}$ its dual sheaf. Then there is a natural exact sequence $[\mathbf{1 9},($ IV, 19$)$, p. 345],

$$
0 \longrightarrow \nu_{X}^{*} \otimes \mathcal{L} \longrightarrow V_{X} \longrightarrow \mathcal{P} \longrightarrow 0
$$

Suppose that $X$ is the complete intersection of hypersurfaces of degrees $d_{1}$, $\ldots, d_{r}$. Then, obviously,

$$
\nu_{X}=\bigoplus_{i=1}^{r} \mathcal{O}_{X}\left(d_{i}\right)
$$

Now, let $\mathcal{L}^{\prime}$ be the pullback to $X^{\prime}$ of the determinant of the universal quotient sheaf on the Grassmannian (that determinant is the line bundle associated to the Plücker embedding). Then

$$
\gamma^{*} \mathcal{L}^{\prime}=\operatorname{det} \mathcal{P}
$$

Hence, the preceding three displays yield the relation

$$
\gamma^{*} \mathcal{L}^{\prime}=\mathcal{L}^{\otimes a} \text { where } a:=\sum_{i=1}^{r}\left(d_{i}-1\right)
$$

Moreover, clearly (3.3) yields the "Plücker formula":

$$
m d^{\prime}=a^{n} d
$$

where $m:=\operatorname{deg} \gamma, d^{\prime}:=\operatorname{deg} X^{\prime}, n:=\operatorname{dim} X$, and $d:=\operatorname{deg} X\left(\operatorname{so} d=d_{1} \cdots d_{r}\right)$. 
THEOREM 4. Let $X$ be a smooth complete intersection of dimension $n$ and degree at least 2, and assume that the image $X^{\prime}$ of the Gauss map is also smooth. Then

$$
c_{n}(X)=c_{n}\left(X^{\prime}\right)
$$

In fact, that equation holds for any smooth $n$-fold $X^{\prime}$ through which the Gauss map factors.

Proof. In the notation of Setups 1 and 3, the formula will be established by computing the self-intersection number $[D]^{2}$ in two ways. First, because of (3.3), Lemma 2 applies; it yields $[D]^{2}=m^{2}[S]^{2}$. Hence (1.2) and (3.1) yield

$$
[D]^{2}=m^{2} c_{n}(X)
$$

On the other hand, since $\sigma^{\prime}$ is an embedding and $X^{\prime}$ is smooth,

$$
\nu_{\sigma^{\prime}}={\sigma^{\prime *}}_{Y^{\prime}}-\tau_{X}=\sigma^{*} \eta^{*} \tau_{\pi^{\prime}}+{\sigma^{\prime}}^{*} \pi^{\prime *} \tau_{X^{\prime}}-\tau_{X}
$$

Now, $\pi^{\prime} \sigma^{\prime}=\gamma$. Moreover, $\eta^{*} \tau_{\pi^{\prime}}=\tau_{\pi}$. So (1.3) and (3.1) yield $\nu_{\sigma^{\prime}}=\gamma^{*} \tau_{X^{\prime}}$. Hence, the self-intersection formula yields

$$
\left[D^{\prime}\right]^{2}=c_{n}\left(\gamma^{*} \tau_{X^{\prime}}\right)
$$

Now, $X$ and $X^{\prime}$ are smooth and $\gamma$ is finite, so $\gamma$ is flat; hence, $[D]^{2}=m\left[D^{\prime}\right]^{2}$ by (1.7). Since $\eta$ is also of degree $m$, the projection formula yields $c_{n}\left(\gamma^{*} \tau_{X^{\prime}}\right)=$ $m c_{n}\left(\tau_{X^{\prime}}\right)$. Hence, (4.2) yields $[D]^{2}=m^{2} c_{n}\left(X^{\prime}\right)$. Therefore, (4.1) yields the assertion.

3. Hypersurfaces and curves. This section treats two cases. In the first, $X$ is a smooth hypersurface of degree at least 2 , and $X^{\prime}$ is the dual hypersurface; that is, $X^{\prime}$ is the image of the Gauss map. Theorem 5 asserts that, if $X^{\prime}$ too is smooth, then $X^{\prime}$ is of the same degree as $X$, with this one exception: $X$ is an odd dimensional quadric in characteristic 2 and $X^{\prime}$ is a hyperplane. If $X$ is a quadric, then more may be asserted, and it is unnecessary to assume that $X^{\prime}$ is smooth; indeed, Proposition 6 asserts this: if $n$ is odd and the characteristic is 2 , then $X^{\prime}$ is a hyperplane and $\gamma$ is purely inseparable of degree 2 ; otherwise, $X^{\prime}$ too is a smooth quadric and $\gamma$ is an isomorphism. Theorem 7 , an immediate consequence of those two results, asserts that the tangent hyperplanes of $X$ contain a common point if and only if $X$ is an odd dimensional quadric in characteristic 2 .

In the second case treated in this section, $C$ is a projective curve with arbitrary singularities. Theorem 8 asserts that if the number $\kappa$ of cusps is strictly less than $2 g-2$, where $g$ is the geometric genus, then the Gauss (rational) map is purely inseparable. Theorem 8 also gives an inequality relating $\kappa$ and $g$ to the separable degree $s$ of the Gauss map and the geometric genus $g^{\prime}$ of its image. Remark 9 gives two variations of the proof of that inequality. Finally, Example 10 gives two examples that illustrate the inequality. 
THEOREM 5. Let $X$ be a smooth hypersurface of dimension $n \geq 1$ and degree $d \geq 2$ in characteristic $p$, and assume that the dual hypersurface $X^{\prime}$ is also smooth. Let $d^{\prime}$ denote the degree of $X^{\prime}$, and $m$ the degree of the Gauss map $\gamma$. Then either (1) $d^{\prime}=d$ and $m=(d-1)^{n}$ or else (2) $n$ is odd, $d=2, d^{\prime}=1$, $m=2, p=2$, and $\gamma$ is purely inseparable. Moreover, if $d \geq 3$, then $p \mid(d-1)$.

Proof. It is well known and easy to show (compare with the first part of the proof of Lemma 12 below) that

$$
c_{n}(X)=P(d) \text { where } P(d):=\sum_{i=0}^{n}(-1)^{i}\left(\begin{array}{c}
n+2 \\
i+2
\end{array}\right) d^{i+1}
$$

Hence, by Theorem 4, $P(d)=P\left(d^{\prime}\right)$. Now, obviously,

$$
(1-d)^{n+2}=1-(n+2) d+P(d) d
$$

Hence the derivative $P^{\prime}(d)$ is given by the formula,

$$
P^{\prime}(d)=-\left[(1-d)^{n+1}((n+1) d+1)-1\right] / d^{2} .
$$

So $(-1)^{n} P^{\prime}(d)>0$ for all real $d \geq 2$. Hence, $(-1)^{n} P(d)$ is strictly increasing in $d$ for $d \geq 2$. Moreover, it is evident from (5.1) that $P(2)=n+2$ if $n$ is even, that $P(2)=n+1$ if $n$ is odd, and that $P(1)=n+1$. Since $P(d)=P\left(d^{\prime}\right)$, therefore either (1) $d^{\prime}=d$ or else (2) $d=2, d^{\prime}=1$, and $n$ is odd.

By (3.4), $m d^{\prime}=d(d-1)^{n}$. Hence, if $d^{\prime}=d$, then $m=(d-1)^{n}$, and if $d=2$ and $d^{\prime}=1$, then $m=2$. Now, if $m>1$, then, by the Monge-Segre-Wallace criterion $[20,(4)$, p. 169], $\gamma$ is inseparable (although perhaps not purely inseparable if $m>p)$, and so $p \mid m$. Hence, if $d \geq 3$, then $p \mid(d-1)$, and if $d=2$ and $d^{\prime}=1$, then $p=2$. Thus the theorem is proved.

Proposition 6. Let $X$ be a smooth quadric hypersurface of dimension $n$. If $n$ is odd and the characteristic $p$ is 2, then the dual hypersurface $X^{\prime}$ is a hyperplane and the Gauss map $\gamma$ is purely inseparable of degree 2; otherwise, $X^{\prime}$ too is a smooth quadric and $\gamma$ is an isomorphism.

Proof. The Gauss map may be computed easily by putting the equation of $X$ in standard form. Alternatively, the assertion may be proved without that computation. Indeed, set $d^{\prime}:=\operatorname{deg} X^{\prime}$ and $m:=\operatorname{deg} \gamma$. By (3.4), $m d^{\prime}=2$; hence, either (a) $d^{\prime}=1$ and $m=2$ or (b) $d^{\prime}=2$ and $m=1$. In Case (a), $X^{\prime}$ is a hyperplane, so smooth; hence, Theorem 5 implies that $n$ is odd, $p=2$, and $\gamma$ is purely inseparable. Conversely, if $n$ is odd and $p=2$, then $m \geq 2$ by [18, Proposition 3.3, p. 221, and $\S 1.2$, p. 214]; so Case (a) obtains.

In Case (b), $X$ is reflexive by the Monge-Segre-Wallace criterion [20, (4), p. 169] because $\gamma$ is birational; so, the dual $X^{\prime \prime}$ of $X^{\prime}$ is equal to $X$. Hence, $X^{\prime}$ cannot be a cone; for otherwise, $X$ would have to be the plane dual to the vertex. Hence, $X^{\prime}$ is a smooth quadric. Since $\gamma$ is birational and, by Lemma 2, finite, therefore $\gamma$ is an isomorphism. 
THEOREM 7. Let $X$ be a smooth hypersurface of degree at least 2. If its tangent hyperplanes contain a common point, then $X$ is an odd dimensional quadric in characteristic 2 , and conversely.

Proof. The tangent hyperplanes contain a common point if and only if the dual hypersurface $X^{\prime}$ is a hyperplane. So the direct assertion follows from the Theorem 5, and the converse from Proposition 6.

THEOREM 8. Let $C$ be an arbitrary (reduced and irreducible) projective curve with $\kappa$ cusps. Let $g$ be its geometric genus, and $g^{\prime}$ that of the image of its Gauss (rational) map. Finally, let $s$ be the separable degree of the Gauss map. Then

$$
2 s\left(g-g^{\prime}\right) \leq(s-1) \kappa .
$$

Moreover, if $\kappa<2 g-2$, then $s=1$; that is, the Gauss map is purely inseparable.

Proof. The proof is, in many ways, similar to that of Theorem 4. Let $X$ be the normalization of $C$. Let $C^{\vee}$ be the image of the Gauss map, and $X^{\prime}$ its normalization, not in its function field $k\left(C^{\vee}\right)$, but in the purely inseparable closure of $k\left(C^{\vee}\right)$ in $k(C)$. Then the Gauss map factors through a separable, finite, and flat map $\gamma: X \rightarrow X^{\prime}$ of degree $s$. Moreover, as $X^{\prime}$ is purely inseparable over $C^{\vee}$, the genus of $X^{\prime}$ is equal to $g^{\prime}$. Since the numerical equivalence class of a 0 -cycle is determined by its degree, Lemma 2 applies; in its notation, but with $m=s$, it asserts that the 1-cycles $[D]$ and $s[S]$ on the surface $Y$ are numerically equivalent. Hence,

$$
[D] \cdot[S]=s[S]^{2} \text { and }[D]^{2}=s^{2}[S]^{2} \text {. }
$$

It is not hard to see (compare [19, pp. 353-354]) that the inclusion of $\mathcal{P}$ in $\mathcal{P}_{X}^{1}(\mathcal{L})$ induces an exact sequence,

$$
0 \longrightarrow \Omega \longrightarrow \Omega_{X}^{1} \longrightarrow \Omega_{X / C}^{1} \longrightarrow 0
$$

Since, by definition, $\kappa$ is equal to the degree of $\Omega_{X / C}^{1}$, therefore

$$
c_{1}(\Omega)=2 g-2-\kappa .
$$

Now, (1.2) and (1.4) yield $[S]^{2}=c_{1}\left(\Omega^{*}\right)$, where $\Omega^{*}$ is the dual sheaf. Hence

$$
[S]^{2}=\kappa-2 g+2 .
$$

Since $\gamma$ is separable, $D$ is reduced; that conclusion was drawn in the paragraph before (1.7). Therefore, $[S]$ appears with multiplicity 1 as a component of $[D]$. Consequently, $([D]-[S]) \cdot[S] \geq 0$. So, $(8.2)$ yields $(s-1)[S]^{2} \geq 0$. Hence, if $s>1$, then $[S]^{2} \geq 0$; whence, (8.4) implies $\kappa \geq 2 g-2$. Thus the second assertion is proved.

Now, $\gamma$ is finite, flat, and of degree $s$, so $[D]^{2}=s\left[D^{\prime}\right]^{2}$ by (1.7). Consequently, (8.2) yields the relation,

$$
\left[D^{\prime}\right]^{2}=s[S]^{2} .
$$


On the other hand, $2 p_{a}\left(D^{\prime}\right)-2=\left[D^{\prime}\right]^{2}-c_{1}\left(\tau_{Y^{\prime}}\right)\left[D^{\prime}\right]$ by the adjunction formula. Obviously, $c_{1}\left(\tau_{Y^{\prime}}\right)\left[D^{\prime}\right]$ is equal to the degree of $\sigma^{\prime *} \tau_{Y^{\prime}}$. Moreover,

$$
\sigma^{\prime *} \tau_{Y^{\prime}}=\sigma^{*} \eta^{*} \tau_{\pi^{\prime}}+\sigma^{\prime *} \pi^{\prime *} \tau_{X^{\prime}}=\sigma^{*} \tau_{\pi}-\gamma^{*} \tau_{X^{\prime}}
$$

Now, $\gamma$ is of degree $s$, so $c_{1}\left(\gamma^{*} \tau_{X^{\prime}}\right)=s\left(2-2 g^{\prime}\right)$. Moreover, (1.2) and (1.3) yield $[S]^{2}=c_{1}\left(\sigma^{*} \tau_{\pi}\right)$. Therefore, (8.5) yields the equation,

$$
2 p_{a}\left(D^{\prime}\right)-2=(s-1)[S]^{2}-s\left(2-2 g^{\prime}\right) .
$$

Plugging (8.4) into that equation and rewriting the result yields this equation:

$$
2\left(p_{a}\left(D^{\prime}\right)-g\right)=(s-1) \kappa-2 s\left(g-g^{\prime}\right) .
$$

Since $\sigma^{\prime}$ is birational, $p_{a}\left(D^{\prime}\right) \geq g$; whence, (8.1) holds. Thus the theorem is proved.

Remark 9. Here are two successive variations on the proof of (8.1). Neither one involves Lemma 2, and each brings the proof another step closer in spirit to Kaji's original proof that a nodal curve of geometric genus at least 2 has a purely inseparable Gauss map.

First, set $d:=c_{1}(\mathcal{L})($ so $d=\operatorname{deg} C)$, and set $k^{\prime}:=c_{1}\left(\mathcal{O}_{Y^{\prime}}(1)\right)$. Then

$$
k^{\prime}\left[D^{\prime}\right]=d \text { and } s k^{\prime 2}=2 d+2 g-2-\kappa .
$$

Indeed, set $k:=c_{1}\left(\mathcal{O}_{Y}(1)\right)$. Then $\eta^{*} k^{\prime}=k$. So $k^{\prime}\left[D^{\prime}\right]=k[D]$, and $s k^{\prime 2}=k^{2}$ as $\eta$ is of degree $s$. So (1.1) yields the first equation of (9.1). As to the second, $k^{2}$ is equal to the second Segre class $s_{2}(\mathcal{P})$; whence, to $2 d+c_{1}(\Omega)$ by $(1.6)$; whence, to $2 d+2 g-2-\kappa$ by $(8.3)$.

Let $f^{\prime}$ be the class of a fiber of $\pi^{\prime}$. Then $f^{\prime 2}=0$ and $f^{\prime} k^{\prime}=1$. Moreover, under the birational map $X \rightarrow D^{\prime}$, the class of a fiber of $\gamma$ corresponds to $f^{\prime}\left[D^{\prime}\right]$; hence, $f^{\prime}\left[D^{\prime}\right]=s$. Now, $\left[D^{\prime}\right]$ is numerically equivalent to $a k^{\prime}+b f^{\prime}$ for some integers $a$ and $b$. Computing $f^{\prime}\left[D^{\prime}\right]$ yields $a=s$. Then computing $k^{\prime}\left[D^{\prime}\right]$ using (9.1) yields $b=\kappa-d+2-2 g$. A third computation yields now $\left[D^{\prime}\right]^{2}=s(\kappa-2 g+2)$. Finally, the argument after (8.5) yields (8.1).

Here is the second variation. Note that $c_{1}\left(\tau_{Y^{\prime}}\right)$ is numerically equivalent to some linear combination $u k^{\prime}+v f^{\prime}$. Since $f^{\prime}$ is of arithmetic genus 0 , the adjunction formula yields $u=2$. Now, let $H^{\prime}$ be the pullback to $Y^{\prime}$ of a general hyperplane of $\mathbf{P}(V)$. Obviously, $H^{\prime}$ is a section of $Y^{\prime} / X^{\prime}$; so $H^{\prime}$ is of genus $g^{\prime}$. Obviously, $\left[H^{\prime}\right]=k^{\prime}$; so the adjunction formula yields $v=2-2 g^{\prime}-k^{\prime 2}$. Finally, instead of computing $c_{1}\left(\tau_{Y^{\prime}}\right)\left[D^{\prime}\right]$ as in the proof of Theorem 8, compute it as an intersection number.

Example 10. Equation (8.6) yields more than the simple inequality (8.1); namely, in (8.1), equality holds if and only if $\sigma^{\prime}: X \rightarrow D^{\prime}$ is an isomorphism. In particular, equality holds if the image of $X$ in the incidence variety is smooth. For instance, equality holds if each singularity of $C$ is an ordinary multiple point or a simple cusp, provided that, when the singularity is a cusp, the characteristic is different from 2. 
Here is an interesting example illustrating the latter case. Given $d$ and $q$, Bayer and Hefez [3, Theorem 6, p. 24] determine the singularities of a plane curve $C$ that is general such that its degree is $d$, its Gauss map is of inseparable degree $q$, and its tangents are concurrent (so $g^{\prime}=0$ ). The multiplicity of $C$ at the point $P$ of concurrence (called the "center" of $C$ ) is equal to the residue $r$ of $d$ modulo $q$; if $r>1$, then $P$ is an ordinary multiple point. If the characteristic is different from 2 , then there are $s(d+r-2)$ remaining multiple points, and each is a simple cusp; moreover, $g=\frac{1}{2}(s-1)(d+r-2)$. Hence $2 g s=(s-1) \kappa$.

Here is a simple example where the inequality in (8.1) is strict (Hefez, pvt. comm., 17 April 1990). Let $C: y=x^{2 q}$, where $q$ is a power of the characteristic $p$, and $p$ is different from 2. Clearly, $C$ has concurrent tangents, and the point of concurrence is not on $C$. Hence, by a formula of Holme and Lluis [21, Formula (3)], $2 q=s i+0$ where $i$ is the inseparable degree of the Gauss map. Hence $s=2$ (and $q=i$ ), but $g^{\prime}=0$ and $g=0$. Thus $2 s\left(g-g^{\prime}\right)=0$, but $(s-1) \kappa>0$.

4. Surfaces. This section gives two criteria for a smooth surface $X$ to have a purely inseparable Gauss map. The first criterion, given in Proposition 11, is relatively abstract. The second criterion, given in Theorem 13, is more concrete: $X$ need only be a complete intersection of degree at least 2 . Theorem 13 is proved by checking the criterion of Proposition 11 with the aid of Lemma 12. There are, however, five exceptional cases, which must be checked directly.

Proposition 11. Let $X$ be a smooth projective surface of degree $d$ at least 2. Set $h:=c_{1}\left(\mathcal{O}_{X}(1)\right)$. Assume that $c_{1}\left(\mathcal{P}_{X}^{1}(1)\right)$ is numerically equivalent to a rational multiple of $h$ and that $c_{1}(X) \leq 4 h$ and $c_{2}(X)>4 d$. Then the Gauss map of $X$ is purely inseparable.

Proof. Let $X^{\vee}$ be the image of the Gauss map, and $X^{\prime}$ the normalization of $X^{\vee}$ in the purely inseparable closure of its function field in the field of $X$. Then the Gauss map factors through a separable map $\gamma: X \rightarrow X^{\prime}$ of degree $s$. Use the notation of Setups 1 and 3, but with $m=s$. Recall from the end of Setup 1 that, as $\gamma$ is separable, $D$ is reduced. Finally, note that $c_{1}\left(\gamma^{*} \mathcal{O}_{X^{\prime}}(1)\right)$ is numerically equivalent to a rational multiple of $h$ because $c_{1}\left(\mathcal{P}_{X}^{1}(1)\right)$ is so by hypothesis and because of (3.2).

Let $\tilde{\pi}: \widetilde{Y} \rightarrow Y$ be the blowup along $S$. Let $\widetilde{S}$ be the strict transform of $D$, let $E$ be the exceptional divisor $\tilde{\pi}^{-1} S$, and set

$$
R:=\widetilde{S} \cap E
$$

Note that $\widetilde{S}$ is reduced because $D$ is. Hence, $R$ is a divisor on $\widetilde{S}$. Moreover, because $D$ is reduced, the 2-cycle $[S]$ appears with multiplicity 1 as a component of the 2 -cycle $[D]$; hence, the direct image $\tilde{\pi}_{*}[\widetilde{S}]$ is equal to the difference $[D]-[S]$. Hence, Lemma 2 yields the relation

$$
\widetilde{\pi}_{*}[\widetilde{S}]=(s-1)[S]
$$

modulo numerical equivalence on $Y$. 
The assertion is, in other words, that $s=1$, and it will be proved by computing the refined intersection number $[S] \cdot_{Y}[\widetilde{S}]$ in two ways. First, consider the corresponding Cartesian diagram:

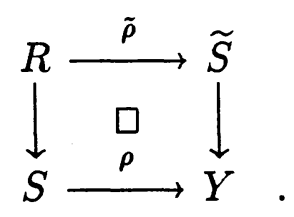

By the compatibility of pullback with proper pushforth, $[S] \cdot Y[\widetilde{S}]$ is equal to $[S] \cdot Y \tilde{\pi}_{*}[\widetilde{S}]$. Hence, $(11.2),(1.2)$ and (3.1) yield

$$
[S] \cdot Y[\widetilde{S}]=(s-1) c_{2}(X) .
$$

On the other hand, the excess-intersection formula [5, Thm. 6.3, p. 102] yields

$$
[S] \cdot Y[\widetilde{S}]=c_{1}\left(\nu_{\rho}\right)[R]-c_{1}\left(\nu_{\tilde{\rho}}\right)[R] .
$$

Now, $\nu_{\rho}=\nu_{\sigma}$. So (3.1) yields $c_{1}\left(\nu_{\rho}\right)=c_{1}(X)$. Hence, (11.1) yields

$$
[S] \cdot Y[\widetilde{S}]=c_{1}(X)[R]-[E]^{2}[\widetilde{S}] .
$$

The term $[E]^{2}[\widetilde{S}]$ will now be evaluated.

Consider the natural map associated to (1.5) (see [19, (V, B), pp. 368-370]):

$$
\lambda: \tilde{Y} \longrightarrow L \text { where } L:=\mathbf{P}(\Omega \otimes \mathcal{L}) .
$$

Note that $L$ and $E$ are isomorphic schemes, but they have different fundamental sheaves $\mathcal{O}_{L}(1)$ and $\mathcal{O}_{E}(1)$; indeed, $E:=\mathbf{P}(\Omega)$ because of (3.1). Geometrically, $\lambda$ is given as follows. Let $x \in X$. Then $\pi^{-1} x$ is equal to the embedded tangent plane $T_{x} X$, and $\tilde{\pi}^{-1} \pi^{-1} x$ is equal to the blowup of $T_{x} X$ at $x$. Finally, $\lambda \mid \tilde{\pi}^{-1} \pi^{-1} x$ is equal to the retraction onto the exceptional divisor induced by the projection of $T_{x} X$ from $x$.

Since the ideal of $S$ is equal to the image of the map (1.6), $S$ is equal to the scheme of zeros of the composition,

$$
\pi^{*}(\Omega \otimes \mathcal{L}) \longrightarrow \pi^{*} \mathcal{P} \longrightarrow \mathcal{O}_{Y}(1)
$$

So $\lambda$ corresponds to the 1-quotient $\mathcal{O}_{\widetilde{Y}}(1) \otimes \tilde{\pi}^{*} \mathcal{O}_{Y}(1)$ of $\tilde{\pi}^{*} \pi^{*}(\Omega \otimes \mathcal{L})$; that is, that 1 -quotient is equal to $\lambda^{*} \mathcal{O}_{L}(1)$. Since $\mathcal{O}_{\widetilde{Y}}(1)$ is the ideal of $E$, the class $[E]$ is given by the formula,

$$
[E]=\tilde{\pi}^{*} k-\lambda^{*} l \text { where } k:=c_{1}\left(\mathcal{O}_{Y}(1)\right) \text { and } l:=c_{1}\left(\mathcal{O}_{L}(1)\right) \text {. }
$$

To (11.5), apply the identity $z^{2}=y^{2}-x^{2}+2 x z$ where $z=x-y$ :

$$
[E]^{2}=\lambda^{*} l^{2}-\tilde{\pi}^{*} k^{2}+2 \tilde{\pi}^{*} k[E]
$$


Therefore, (11.2) and (11.1) and the projection formula yield

$$
[E]^{2}[\widetilde{S}]=\lambda^{*} l^{2}[\widetilde{S}]-(s-1) k^{2}[S]+2 k \widetilde{\pi}_{*}[R] .
$$

Since $\mathcal{O}_{Y}(1) \mid S=\mathcal{O}_{X}(1)$ by $(1.1)$, therefore (11.4) becomes

$$
[S] \cdot Y[\widetilde{S}]=\left(c_{1}(X)-2 h\right) \widetilde{\pi}_{*}[R]+(s-1) d-\lambda^{*} l^{2}[\widetilde{S}] .
$$

To evaluate the term $\lambda^{*} l^{2}[\widetilde{S}]$, consider the natural map,

$$
\tau: L \longrightarrow \operatorname{Grass}^{2}(V) .
$$

Geometrically, it is defined by sending a tangent line to $X$ at a point $x$, viewed in the tangent plane $T_{x} X$, to the same line viewed in the ambient projective space $\mathbf{P}(V)$. Algebraically, $\tau$ corresponds to the 2-quotient $\mathcal{Q}$ of $V_{L}$ arising from the canonical surjection $V_{L} \rightarrow \mathcal{P}_{L}$ and a certain surjection $\mathcal{P}_{L} \rightarrow \mathcal{Q}$; the latter is defined by this pushout diagram:

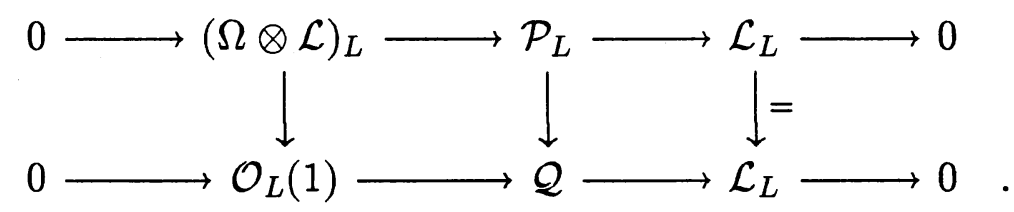

Set $g:=c_{1}(\mathcal{Q})$. Then the bottom row above yields $g=l+h$. Hence,

$$
\begin{aligned}
\lambda^{*} l^{2} & =\left(\lambda^{*} g-\tilde{\pi}^{*} \pi^{*} h\right)^{2} \\
& =\lambda^{*} g^{2}-2 \lambda^{*} g \tilde{\pi}^{*} \pi^{*} h+\tilde{\pi}^{*} \pi^{*} h^{2} \\
& =\lambda^{*} g^{2}-2 \lambda^{*} l \tilde{\pi}^{*} \pi^{*} h-\tilde{\pi}^{*} \pi^{*} h^{2} .
\end{aligned}
$$

Therefore, the projection formula and (11.5) yield

$$
\lambda^{*} l^{2}[\widetilde{S}]=g^{2} \tau_{*} \lambda_{*}[\widetilde{S}]-2\left(\tilde{\pi}^{*} k-[E]\right)\left(\tilde{\pi}^{*} \pi^{*} h\right)[\widetilde{S}]-\left(\tilde{\pi}^{*} \pi^{*} h\right)^{2}[\widetilde{S}] .
$$

Hence, (11.1) and the projection formula yield

$$
\lambda^{*} l^{2}[\widetilde{S}]=g^{2} \tau_{*} \lambda_{*}[\widetilde{S}]-\left(2 k+\pi^{*} h\right) \pi^{*} h \widetilde{\pi}_{*}[\widetilde{S}]+2 \widetilde{\pi}^{*} \pi^{*} h^{2}[R] .
$$

Since $\mathcal{O}_{Y}(1) \mid S=\mathcal{O}_{X}(1)$ by (1.1), therefore (11.2) yields

$$
\lambda^{*} l^{2}[\widetilde{S}]=g^{2} \tau_{*} \lambda_{*}[\widetilde{S}]-3(s-1)\left(\pi^{*} h\right)^{2}[S]+2 \tilde{\pi}^{*} \pi^{*} h^{2}[R] .
$$

Since $\left(\pi^{*} h\right)^{2}[S]=d$, therefore $(11.6)$ becomes

$$
[S] \cdot Y[\widetilde{S}]=\left(c_{1}(X)-4 h\right) \widetilde{\pi}_{*}[R]+4(s-1) d-g^{2} \tau_{*} \lambda_{*}[\widetilde{S}] .
$$

Finally, combining that equation with (11.3) yields

$$
(s-1)\left(c_{2}(X)-4 d\right)=\left(c_{1}(X)-4 h\right) \tilde{\pi}_{*}[R]-g^{2} \tau_{*} \lambda_{*}[\widetilde{S}] .
$$

Since $g$ is very ample and, by hypothesis, $c_{1}(X) \leq 4 h$, the right side of that equation is at most 0 . On the other hand, by hypothesis, $c_{2}(X)>4 d$; so the left side is at least 1 if $s>1$. Therefore, $s=1$, as asserted. 
LEMMA 12. Let $X$ be a smooth projective surface. Assume that $X$ is a complete intersection of hypersurfaces of degrees $d_{1}, \ldots, d_{r}$ and that $d_{i} \geq 2$ for all $i$.

(1) Set $h:=c_{1}\left(\mathcal{O}_{X}(1)\right)$. Then $c_{1}(X)=$ ch for some $c \leq 2$.

(2) Set $d:=d_{1} \cdots d_{r}$. Then $c_{2}(X)>4 d$ except in these five cases:

$$
\left(d_{1}, \ldots, d_{r}\right)=(2),(3),(2,2),(2,3),(2,2,2) .
$$

In those five cases, respectively $c_{1}(X)=2 h, h, h, 0,0$.

Proof. As is well known, it follows from the Euler sequence and the tangent sheaf-normal sheaf sequence that

$$
\begin{aligned}
c(X) & =(1+h)^{r+3}\left(1+d_{1} h\right)^{-1} \cdots\left(1+d_{r} h\right)^{-1} \\
& =\left(1+(r+1) h+\left(\begin{array}{c}
r+3 \\
2
\end{array}\right) h^{2}\right)\left(1-d_{1} h+d_{1} h^{2}\right) \cdots\left(1-d_{r} h+d_{r} h^{2}\right) .
\end{aligned}
$$

Hence $c_{1}(X)=c h$ where $c=\left(r+3-\sum d_{i}\right)$. Since $d_{i} \geq 2$, therefore $c \leq 3-r \leq 2$. Moreover, the last assertion follows.

Obviously $h^{2}=d$. So the expression for $c(X)$ above yields

$$
c_{2}(X)=Q\left(d_{1}, \ldots, d_{r}\right) d
$$

where

$$
Q\left(d_{1}, \ldots, d_{r}\right):=\left(\begin{array}{c}
r+3 \\
2
\end{array}\right)-(r+3) \sum_{i=1}^{r} d_{i}+\sum_{i<j} d_{i} d_{j}+\sum_{i=1}^{r} d_{i}^{2} .
$$

Clearly

$$
Q(2, \ldots, 2)=\left(\begin{array}{c}
r+3 \\
2
\end{array}\right)-(r+3)(2 r)+\left(\begin{array}{c}
r \\
2
\end{array}\right) 4+4 r=\frac{1}{2} r^{2}-\frac{3}{2} r+3 .
$$

Therefore, $Q(2, \ldots, 2)>4$ if $r>3$. Moreover,

$$
Q(2)=2, Q(2,2)=2 \text {, and } Q(2,2,2)=3 .
$$

To deal with the case of arbitrary $d_{i}$, note that

$$
\begin{aligned}
Q\left(d_{1}, \ldots, d_{r}+1\right)-Q\left(d_{1}, \ldots, d_{r}\right) & =-(r+3)+\sum_{1}^{r-1} d_{i}+2 d_{r}+1 \\
& =\sum_{1}^{r}\left(d_{i}-1\right)+\left(d_{r}-2\right) .
\end{aligned}
$$

Since $d_{i} \geq 2$, therefore

$$
Q\left(d_{1}, \ldots, d_{r}+1\right)-Q\left(d_{1}, \ldots, d_{r}\right) \geq r
$$

and equality holds if and only if $d_{i}=2$ for all $i$. Hence, by (12.1),

$$
Q(3)=3, \text { and } Q(2,3)=4 \text {. }
$$

Finally, $Q\left(d_{1}, \ldots, d_{r}\right)$ is obviously symmetric in $d_{1}, \ldots, d_{r}$. So, it follows from (12.2) by induction on $\sum d_{i}$ that $Q\left(d_{1}, \ldots, d_{r}\right)>4$ except in the five cases given in (12.1) and (12.3). Thus the lemma is proved. 
TheOREm 13. Let $X$ be a smooth projective surface of degree at least 2. Assume that $X$ is a complete intersection. Then its Gauss map $\gamma$ is purely inseparable.

Proof. Say that $X$ is the complete intersection of hypersurfaces $F_{1}, \ldots, F_{r}$ of degrees $d_{1}, \ldots, d_{r}$. Obviously, we may assume that $d_{i} \geq 2$ for all $i$. Then by (3.3), by Lemma 12, and by Proposition $11, \gamma$ is purely inseparable, except possibly in the five cases listed in (2) of the Lemma 12. These five cases will now be handled directly.

Suppose that $\gamma$ is not purely inseparable, and let $x$ be a general point of $X$. Then the tangent plane $T$ at $x$ makes a second contact, say at $y$ with $y \neq x$. Consider the line $L$ joining $x$ and $y$. If $T \subset F_{i}$, then $L \subset F_{i}$. If $T \not \subset F_{i}$, then $C_{i}:=T \cap F_{i}$ is a plane curve of degree $d_{i}$, which is singular at both $x$ and $y$. If $d_{i}=2$, then $C_{i}$ must be $2 L$. If $d_{i}=3$, then $C_{i}$ must be the sum of $L$ and a conic. Thus, again, $L \subset F_{i}$. Hence, in each of the five cases, $L$ lies in the intersection of the $F_{i}$, which is $X$. Now, the value of $c_{1}(X)$ is given in (2) of the last lemma; whence, the adjunction formula yields respectively

$$
[L]^{2}=0,-1,-1,-2,-2 \text {. }
$$

In the first of the five case, $X$ is a quadric surface in $\mathrm{P}^{3}$. Then $2 L$ is a plane section with self-intersection 0 , an impossibility. In the remaining four cases, consider the scheme $H$ of lines on $X$. The point of $H$ representing $L$ must be isolated. Indeed, otherwise, $X$ would contain a line $M$ distinct from $L$, but algebraically equivalent to it; however, then

$$
0 \leq[M] \cdot[L]=[L]^{2}<0
$$

Now, $H$ is a subscheme of the Grassmanian of lines in $\mathbf{P}^{r+2}$; so $H$ has only finitely many isolated points. Thus $L$ is one of finitely many lines. Consequently, $x$, which lies on $L$, cannot be a general point of $X$, contrary to the way it was chosen. Thus the assertion holds.

5. The Fermat surface. In this section, the Fermat surface is characterized as the unique smooth surface of degree at least 3 in $\mathbf{P}^{3}$ whose dual surface is also smooth. This result, Theorem 14, is the deepest of the paper; its proof relies on much of what has been proved so far.

Theorem 14. Let $X$ be a smooth closed surface of degree $d$ at least 3 in $\mathbf{P}^{3}$, and let $X^{\prime}$ be the dual surface. If $X^{\prime}$ is also smooth, then $X$ is equal to the Fermat surface given, in a suitable coordinate system, by the equation $\sum_{i=0}^{3} x_{i}^{q+1}=0$ with $q=p^{e}$ where $p$ is the characteristic and $e \geq 1$. Moreover, the converse holds.

Proof. Suppose $X$ is the Fermat surface. Then the Gauss map is obviously equal to the Frobenius $q$ th power map. So $X^{\prime}$ is equal to $X$; in particular, $X^{\prime}$ is smooth. Thus the converse holds.

Assume that $X^{\prime}$ is smooth. Let $m$ be the degree of the Gauss map $\gamma: X \rightarrow X^{\prime}$. Then Theorem 5 says that $d$ is also the degree of $X^{\prime}$ and that $m=(d-1)^{2}$. 
Moreover, Theorem 13 says that $\gamma$ is purely inseparable. Hence $m \geq 4$, and $m$ is an even power of $p$; say, $m=p^{2 e}$. Then

$$
d-1=p^{e} \text { and } e \geq 1 \text {. }
$$

Let $C^{\prime}$ be a general plane section of $X^{\prime}$. Then $C^{\prime}$ is a smooth, irreducible plane curve of degree $d$. Consider $D:=\gamma^{-1} C^{\prime}$. By (3.3),

$$
\mathcal{O}_{X}(D)=\mathcal{O}_{X}(d-1)
$$

By Lemma 2, $\gamma$ is finite. Since $\gamma$ is also purely inseparable and its target is normal, it is injective. (Indeed, let $A$ be a normal domain, let $B$ be a domain that contains $A$, is a finitely generated $A$-module, and whose fraction field is purely inseparable of degree $q$ over the fraction field $K$ of $A$. If $P$ and $Q$ are two prime ideals of $B$ such that $P \cap A=Q \cap A$, then $P=Q$. In fact, $P$ (resp. $Q$ ) is equal to the set $S$ of elements $b \in B$ such that $b^{q} \in P \cap A$. For, obviously, $S \subseteq P$, and conversely, if $b \in P$, then $b^{q}$ is in $P \cap K$ and is integral over $A$, so $b^{q} \in P \cap A$, so $P \subseteq S$.) Since $\gamma$ is injective, surjective, and closed, it is a homeomorphism. Hence $D$ is irreducible. Let $C$ be its reduction. Then, for some $l$,

$$
[D]=l[C] \text {. }
$$

Now, (14.2) implies that $[D]^{2}=(d-1)^{2} d$. Therefore, (14.1) and (14.3) yield

$$
l^{2}[C]^{2}=p^{2 e} d \text {. }
$$

Consider the restriction $C \rightarrow C^{\prime}$. Since it is injective, it is purely inseparable, say of degree $p^{f}$. So, if a divisor is pulled back from $C^{\prime}$ to $C$, then its degree is multiplied by $p^{f}$. Hence $[D] \cdot[C]=p^{f}\left[C^{\prime}\right]^{2}$, or $l[C]^{2}=p^{f} d$. Dividing the latter equation into Equation (14.4) yields

$$
l=p^{2 e-f} .
$$

So $l$ is prime to $d$ because of (14.1). So (14.4) implies that $l$ divides $p^{e}$. Hence $2 e-f \leq e$, or

$$
f \geq e
$$

Now, (14.1) and (14.2) yield $\operatorname{deg} D=p^{e} d$. So (14.3) and (14.5) yield

$$
\operatorname{deg} C=(\operatorname{deg} D) / l=\left(p^{e} d\right) / p^{2 e-f}=p^{f-e} d .
$$

Let $g$ denote the geometric genus of $C$. Then $g$ is also the geometric genus of $C^{\prime}$, because $C \rightarrow C^{\prime}$ is purely inseparable. Since $C^{\prime}$ is a smooth plane curve of degree $d$, therefore and by (14.1)

$$
2 g-2=d(d-3)=\left(p^{e}-2\right) d .
$$

Let $p_{a}$ denote the arithmetic genus of $C$. Let $H$ be a hyperplane section of $X$. Then the adjunction formula and (14.2) and (14.3) yield

$$
2 p_{a}-2=((d-4)[H]+[C]) \cdot[C]=\left(d-4+\frac{d-1}{l}\right)\left(\frac{d-1}{l}\right)[H]^{2} .
$$


Since $[H]^{2}=d$, therefore (14.1) and (14.5) yield

$$
2 p_{a}-2=\left(p^{e}-3+p^{f-e}\right) p^{f-e} d .
$$

Let $\bar{C}$ be the normalization of $C$, and $\mathbf{G}$ the Grassmannian of lines in $\mathbf{P}^{3}$. Consider the Gauss map of $C$,

$$
\delta: \bar{C} \stackrel{\hookrightarrow}{G} .
$$

The class $d^{\vee}$ of $C$ is defined by the formula,

$$
d^{\vee}:=(\operatorname{deg} \delta)(\operatorname{deg} \delta \bar{C}),
$$

where the degree $\operatorname{deg} \delta \bar{C}$ is taken with respect to the Plücker embedding of $\mathbf{G}$. If $\kappa$ denotes the number of cusps of $C$, then $d^{\vee}$ is given by the Pohl Plücker formula [19, (IV,41), p. 355] (compare with (9.1)):

$$
d^{\vee}=2 \operatorname{deg} C+(2 g-2)-\kappa .
$$

Rewriting the formula using (14.7) and (14.8) yields

$$
d^{\vee}=2 p^{f-e} d+\left(p^{e}-2\right) d-\kappa .
$$

There is a point $P$ common to all the tangent planes of $X$ at the points of $C$; in fact, $P$ is the point dual to the plane defining $C^{\prime}$. As that plane is general, $P$ is a general point of $\mathbf{P}^{3}$. In particular, $P$ lies off $X$, so off $C$. It will now be proved that there is a simple point of $C$ whose tangent line does not contain $P$.

Suppose to the contrary that $P$ lies on the tangent line at every simple point. Then the Gauss map $\delta$ of $C$ may be identified with the projection from $P$. Hence, since $P$ lies off $C$, the class $d^{\vee}$ is equal to the $\operatorname{degree} \operatorname{deg} C$. Hence (14.10) yields

$$
\operatorname{deg} C=\kappa+2-2 g
$$

Let $C_{1}^{\prime}$ be a second general plane section of $X^{\prime}$, and let $C_{1}$ and $P_{1}$ denote its reduced preimage and dual point. The integer $l_{1}$ corresponding to $l$ is equal to $l$, because $\gamma^{-1} C^{\prime}$ deforms continuously into $\gamma^{-1} C_{1}^{\prime}$ in an algebraic family, and the reduction of the total space is the total space of a family in which $C$ deforms to $C_{1}$. By the same token, the singular locus of $C$ deforms continuously into that of $C_{1}$. Hence, since the family has no base points, neither $C$ nor $C_{1}$ contains a singular point of the other by a count of constants (the set of pairs of reduced preimages such that one contains a singular point of the other is a closed subset of positive codimension in the set of all pairs). Since $P$ and $P_{1}$ are independent general points of $\mathbf{P}^{3}$, the line they determine is not tangent to either $C$ or $C_{1}$. However, that line is the only possible common tangent. Therefore, $C$ and $C_{1}$ intersect transversally.

The intersection $C \cap C_{1}$ is carried bijectively by $\gamma: X \rightarrow X^{\prime}$, which is a homeomorphism, onto the intersection $C^{\prime} \cap C_{1}^{\prime}$. The latter consists of $d$ distinct 
points because $X^{\prime}$ is of degree $d$. Hence, since $C$ and $C_{1}$ intersect transversally, $d=[C] \cdot\left[C_{1}\right]$. On the other hand, the reasoning that gave (14.9) yields

$$
[C] \cdot\left[C_{1}\right]=\left(\frac{d-1}{l}\right)^{2}[H]^{2}=p^{2(f-e)} d
$$

Therefore, $f-e=0$. Hence, (14.7) yields $\operatorname{deg} C=d$. Moreover, (14.8) and (14.9) imply that the geometric genus of $C$ is equal to its arithmetic genus; whence, $C$ is smooth and, in particular, $\kappa=0$. Therefore, (14.12) yields

$$
2 \leq d=\operatorname{deg} C=2-2 g \text {. }
$$

Consequently, $d=2$ (and $g=0$ ). However, by hypothesis, $d \geq 3$. Thus $P$ does not lie on the tangent line at every simple point of $C$.

Let $S$ be the subvariety of the Grassmannian $\mathbf{G}$ of lines through $P$, and consider the map $\beta$ from $\mathbf{G}-S$ to the dual $\mathbf{P}^{3}$ that sends a line $L$ to the plane spanned by $L$ and $P$. Since $P$ does not lie on the tangent line at every simple point of $C$, clearly $\beta$ carries $(\delta \bar{C})-S$ onto an open subset of $C^{\prime}$. So

$$
\operatorname{deg} \delta \bar{C} \geq(\operatorname{deg} \beta)\left(\operatorname{deg} C^{\prime}\right)=(\operatorname{deg} \beta) d
$$

(and the inequality is an equality if and only if $P$ lies on no tangent line of $C$ ). It is not hard to check using the sheaves of principal parts that the composition $\beta \delta: \bar{C} \rightarrow C^{\prime}$ is equal to the composition of the normalization map $\bar{C} \rightarrow C$ with the restriction $C \rightarrow C^{\prime}$ of $\gamma$. Since that restriction is of degree $p^{f}$, therefore

$$
d^{\vee} \geq(\operatorname{deg} \delta)(\operatorname{deg} \beta) d=p^{f} d .
$$

Relations (14.11) and (14.13) yield $p^{f} \leq 2 p^{f-e}+p^{e}-2-(\kappa / d)$, or

$$
p^{f} \leq p^{f}\left(\frac{2}{p^{e}}+\frac{p^{e}}{p^{f}}\right)-2-\frac{\kappa}{d} .
$$

Now, $f \geq e \geq 1$ by (14.6) and (14.1). If $f=e$, then the right hand side above is equal to $p^{f}-\frac{\kappa}{d}$; so $\kappa=0$. If $f>e$, then, since the term in parentheses must be greater than 1 , obviously $p=2$ and $e=1$; in that case too, $\kappa=0$.

Suppose $\kappa=0$; in other words, $C$ has no cusps. Now, $C$ has no nodes; indeed, the composition $\bar{C} \rightarrow C \rightarrow C^{\prime}$ is purely inseparable, so injective because $C^{\prime}$ is normal, and so the normalization map $\bar{C} \rightarrow C$ is injective. Therefore, $C$ is smooth. So its geometric genus $g$ is equal to its arithmetic genus. Hence, (14.8) and (14.9) yield

$$
p^{e}-2=\left(p^{e}-3+p^{f-e}\right) p^{f-e} .
$$

Since $f \geq e$ by (14.6), therefore $f=e$.

Thus, in any case, $f=e$. Hence $C$ is smooth by the preceding two paragraphs, its degree is $d$ by (14.7), and its genus is $d(d-3)$ by (14.8). It follows that $C$ lies in a plane (a general projection $\pi$ maps $C$ birationally onto a plane curve $Z$ with degree $d$, so arithmetic genus $g$; hence, $\pi$ is an isomorphism, whence, $\mathrm{H}^{0}\left(\mathcal{O}_{C}(1)\right)$ 
is of dimension 3). Consequently, $C$ is equal to the section $\Sigma$ of $X$ by the plane it determines; indeed, $[\Sigma]-[C]$ is an effective divisor of degree 0 , so equal to 0 . (Note that, although $(d-1)([C]-[H])$ is linearly equivalent to 0 by $(14.2)$, nevertheless it has to be proved that $[C]-[H]$ is linearly equivalent to 0 .)

By definition, $D$ is the first polar of $X$ with respect to the point $P$ dual to the plane defining $C^{\prime}$, and that plane is general. Moreover, $[D]=p^{e}[C]$ by (14.3) and (14.5), and $C$ is a plane section of $X$ by the preceding paragraph. Therefore, the linear system of all first polars is equal to the $p^{e}$ th multiple of the linear system of plane sections. It follows via a short, fairly simple argument $[2$, (iii) $\Rightarrow$ (iv) and (iv) $\Rightarrow(\mathrm{v})$, pp. 5-6] that $X$ is projectively equivalent to the Fermat surface, as asserted.

\section{REFERENCES}

[1] A. Altman and S. Kleiman, Foundations of a theory of Fano schemes, Compositio Math. 34 (1977), 3-47.

[2] A. Beauville, "Sur les hypersurfaces dont les sections hyperplane sont à module constant," Preprint, spring 1989, to appear in the "Grothendieck Festchrift," Birkhäuser.

[3] V. Bayer and A. Hefez, "Strange curves," Preprint, spring 1989, to appear in Comm. in Algebra.

[4] N. Bourbaki, “Algebra," Hermann; Addison-Wesley, 1974.

[5] W. Fulton, "Intersection theory," Ergebnisse der Mathematik und ihrer Grenzgebiete 3. Folge $\cdot$ Band 2, Springer-Verlag, 1984.

[6] W. Fulton and R. Lazarsfeld, Connectivity and its applications in algebraic geometry, in "Algebraic Geometry, Chicago Circle, 1980," A. Libgober and P. Wagreich (eds.), Lecture Notes in Math. 862, Springer-Verlag, 1981, pp. 26-92.

[7] A. Garcia, "The curves $y^{n}=f(x)$ over finite fields," Preprint, to appear in Arch. Math.

[8] A. Garcia and J. Voloch, "Duality for projective curves," Preprint, impa Série A-085Nov./89.

[9] P. Griffiths and J. Harris, Algebraic geometry and local differential geometry, Ann. Sci. École Norm. Sup. 12 (1979), 355-432.

[10] A. Hefez, Nonreflexive curves, Compositio Math. 69 (1989), 3-35.

[11] A. Hefez and S. Kleiman, Notes on the duality of projective varieties, in "Geometry Today. Roma 1984," E. Arbarello, C. Procesi, E. Strickland (eds.) Prog. Math. 60, Birkhäuser, 1985, pp. 143-184.

[12] A. Hefez and J. Voloch, "Frobenius non-classical curves," Preprint, to appear in Arch. Math.

[13] M. Homma, Funny plane curves in characteristic $p>0$, Comm. Alg. 15 (1987), 14691501.

[14] M. Homma, A souped-up version of Pardini's theorem and its application to funny curves, Compositio Math. 71 (1989), 295-302.

[15] H. Kaji, On the tangentially degenerate curves, J. London Math. Soc. (2) 33 (1986), 430-440.

[16] H. Kaji, On the Gauss maps of space curves in characteristic p, Compositio Math. 70 (1989), 177-197.

[17] H. Kaji, "On the Gauss maps of space curves in characteristic $p$, II," Preprint (J-WASES, July 24, 1989), to appear in Comp. Math.

[18] N. Katz, Pinceaux de Lefschetz: théorème d'existence, SGA 7 II, Exposé XVII, Springer Lecture Notes in Math. 340 (1973), 212-253.

[19] S. Kleiman, The enumerative theory of singularities, in "Real and complex singularities," P. Holm (Ed.), Proc. Conf., Oslo 1976, Sitjhoff \& Noorhoof, 1977, pp. 297-396. 
[20] S. Kleiman, Tangency and duality, in "Proc. 1984 Vancouver Conf. in Algebraic Geometry," J. Carrell, A. V. Geramita, P. Russell (eds.), CMS Conf. Proc. 6, Amer. Math. Soc., 1986, pp. 163-226.

[21] S. Kleiman, "Multiple tangents of smooth plane curves (after Kaji)," Preprint, spring 1989 , to appear in the proceedings of 1988 Sundance conference on algebraic geometry.

[22] R. Piene, Polar classes of singular varieties, Ann. Sci. École Norm. Sup. 11 (1978), 247-276.

[23] J. Rathmann, The uniform position principle for curves in characteristic $p$, Math. Ann. 276 (1978), 565-579.

[24] J. Voloch, "On the geometry of space curves," Preprint, to appear in the Proceedings of the Tenth School of Algebra, Vitoria, Brazil, 1989.

[25] A. Wallace, Tangency and duality over arbitrary fields, Proc. London Math. Soc. (3) 6 (1956), 321-342.

Mathematics Department, Room 2-278, M.I.T., Cambridge, MA 02139, USA

Matematisk Institutt, Universitetet i Oslo, Box 1053, Blindern, 0316 Oslo 3, Norway 